\title{
Tunneling Magnetoresistance in a Two-Layered $\mathrm{La}_{2-2 x} \mathrm{Ca}_{1+2 x} \mathrm{Mn}_{2} \mathrm{O}_{7}$ Polycrystal
}

\author{
Tie-Jun Zhou, Z. Yu, and Y. W. Du \\ Department of Physics and National Laboratory of Solid State Microstructures, P. O. Box 2123, \\ Nanjing University, Nanjing 210093, China \\ Reprint requests to T. J. Z.; E-mail: scmb@netra.nju.cn \\ Z. Naturforsch. 54 a, 695-698 (1999); received August 20, 1999 \\ By the sol-gel method, a polycrystal bulk of two-layered $\mathrm{La}_{2-2 x} \mathrm{Ca}_{1+2 x} \mathrm{Mn}_{2} \mathrm{O}_{7}$ with $x=0.2$ was \\ successfully synthesized. A high sensitive low-field TMR was found at low temperature.
}

PACS numbers: $75.30 . \mathrm{Kz}, 72.15 . \mathrm{Gd}$

\section{Introduction}

The discovery of giant magnetoresistance (GMR) in magnetic multilayer films [1 - 2] and granular solids [3 - 4] has triggered considerable interest in relating the spin-dependent transport phenomena from the viewpoint of both the underlying physics and their immediate application to magnetic storage and sensor technology. A number of the GMR structures have so far been studied, such as magnetic multilayers with antiferromagnetic coupling, spin values [5], granular solids, and tunneling structures [6]. Of all the GMR structures, the tunneling ones are currently investigated extensively because of their large magnetoresistance (MR) ratio in a very low field [7 - 9]. The MR ratio in tunneling structures is dependent on the spin polarization and the relative orientation of the magnetizations in the magnetic layers. Recently, a large tunneling magnetoresistance (TMR) as high as $83 \%$ was observed at low fields of only tens of Oe in an epitaxial tunnel junction with the form $\mathrm{La}_{0.67} \mathrm{Sr}_{0.33} \mathrm{MnO}_{3} / \mathrm{SrTiO}_{3} / \mathrm{La}_{0.67} \mathrm{Sr}_{0.33} \mathrm{MnO}_{3}$, and this high MR value had been attributed to the almost full spin polarization in the manganite layers [10]. Also, a large low-field MR has been examined in polycrystalline manganites (TMR in granular solids) [11].

In fact, there exist natural tunneling structures, and one of them is layered perovskite manganite. This system intrinsically includes magnetic multilayers in its crystal structure (Figure 1). The simplest view

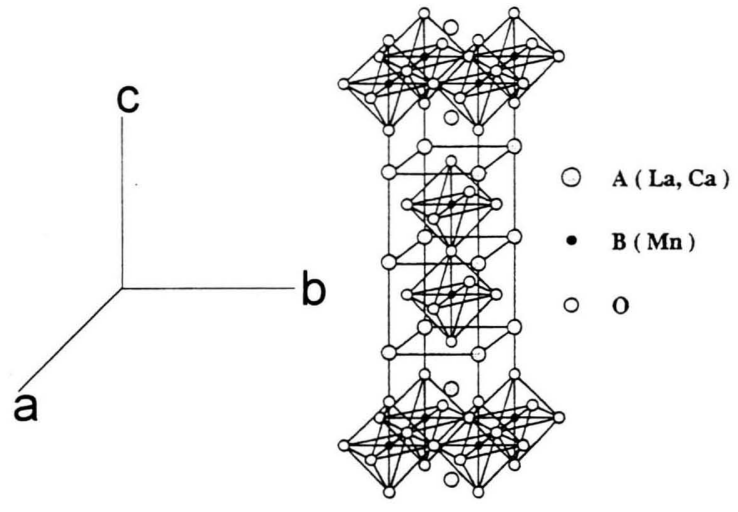

Fig. 1. Crystal structure of $\mathrm{La}_{2-2 x} \mathrm{Ca}_{1+2 x} \mathrm{Mn}_{2} \mathrm{O}_{7}$ with the $\mathrm{Sr}_{3} \mathrm{Ti}_{2} \mathrm{O}_{7}$-type structure.

of layered manganite is a stack of ferromagneticmetallic sheets consisting of $\mathrm{MnO}_{2}$ bilayers which are respectively separated by the $(\mathrm{La}, \mathrm{M})_{2} \mathrm{O}_{2}$ layers which work as nonmagnetic insulating layers. That is to say the layered manganite crystal forms a naturally infinite array of ferromagnetic-metal / insulating / ferromagnetic-metal junctions. In this simple view, the nearly fully spin-polarized electrons' tunneling between the two $\mathrm{MnO}_{2}$ bilayers should give a large low-field TMR. Very recently it was reported the TMR in two-layered perovskite single crystals can attain as large as $240 \%$ in low field at low temperature and this value can be drastically enhanced up to $\sim 4000 \%$ by applying a pressure of $\sim 10$ kbar at 


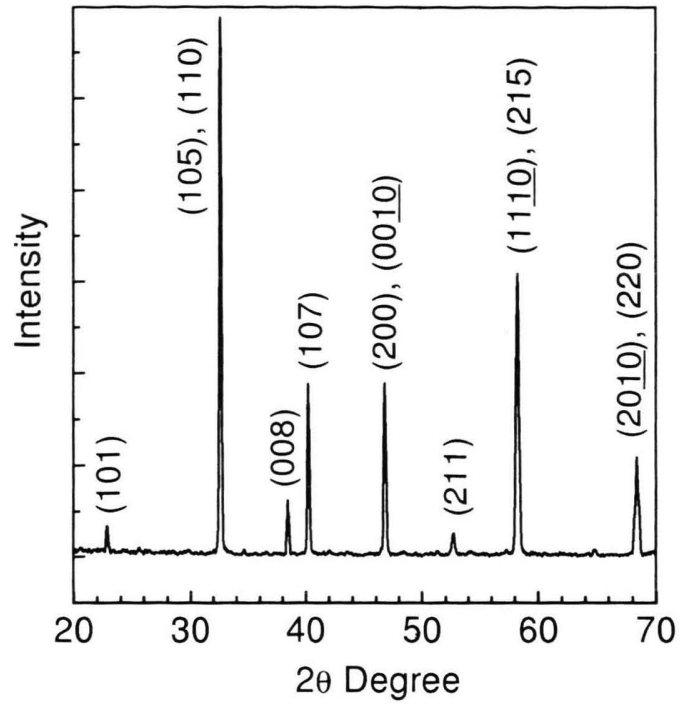

Fig. 2. The XRD pattern for the $\mathrm{La}_{2-2 x} \mathrm{Ca}_{1+2 x} \mathrm{Mn}_{2} \mathrm{O}_{7}$ with $x=0.2$.

$4.2 \mathrm{~K}[12,13]$. All of the past studies on layered perovskites, to the best knowledge of us, were focused on single crystals and films, and not on polycrystal bulks. In this paper, a polycrystal bulk of two-layered $\mathrm{La}_{2-2 x} \mathrm{Ca}_{1+2 x} \mathrm{Mn}_{2} \mathrm{O}_{7}$ with $x=0.2$ was successfully synthesized, and a high-sensitive low-field TMR was discovered at low temperature.

The polycrystalline samples of $\mathrm{La}_{2-2 x} \mathrm{Ca}_{1+2 x^{-}}$ $\mathrm{Mn}_{2} \mathrm{O}_{7}$ with $x=0.2$ were prepared by the sol-gel method in order to obtain well-mixed reagents. Stoichiometric amounts of $\mathrm{La}_{2} \mathrm{O}_{3}, \mathrm{CaCO}_{3}$ and $\mathrm{Mn}\left(\mathrm{NO}_{3}\right)_{2}$ were dissolved in concentrated nitric acid. Afterwards, citric acid was added, the solution was heated at around $350 \mathrm{~K}$ for over two days, giving a gel, followed by decomposition at $470 \mathrm{~K}$ and calcination in air at $1470 \mathrm{~K}$ for 10 hours. The calcined material was ground and pressed into round disks. And final sintering process step was carried out at $1670 \mathrm{~K}$ in air for 30 hours, followed by cooling of the furnace. X-ray diffraction (XRD) and Scanning Electron Microscopy (SEM) were used to characterize the structure of the sample. The electrical resistance and MR were measured as function of temperature and applied field by a standard four-point technique. The MR ratio is defined as $\Delta R / R_{0}=\left(R_{H}-R_{0}\right) / R_{0}$, where $\mathrm{R}_{H}$ and $\mathrm{R}_{0}$ are the resistance at applied field $H$ and zero-field resistance, respectively.

The XRD pattern for the $\mathrm{La}_{2-2 x} \mathrm{Ca}_{1+2 x} \mathrm{Mn}_{2} \mathrm{O}_{7}$ with $x=0.2$ is presented in Figure 2. The diffraction

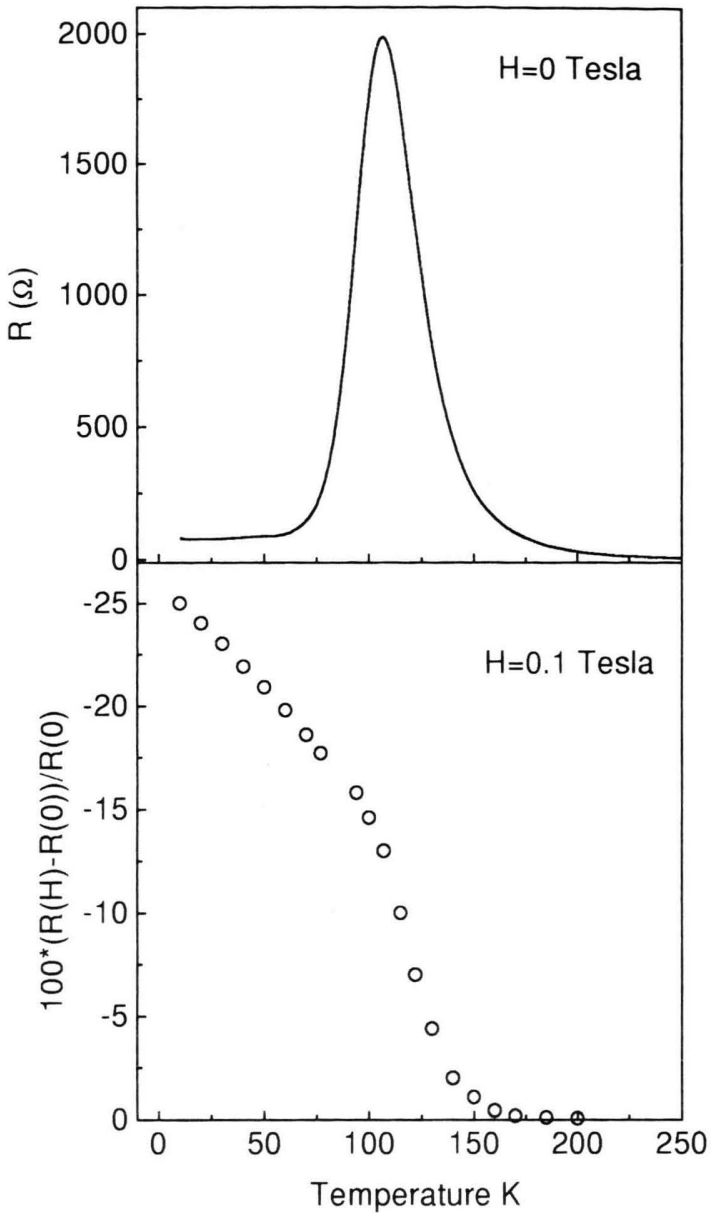

Fig. 3. The temperature dependences of the resistance $R$ without field and the magnetoresistance MR with 0.1 Tesla field for $\mathrm{La}_{2-2 x} \mathrm{Ca}_{1+2 x} \mathrm{Mn}_{2} \mathrm{O}_{7}$.

peaks are indexed with respect to the $\mathrm{Sr}_{3} \mathrm{Ti}_{2} \mathrm{O}_{7}$ type structure. The space group is $\mathrm{I} 4 / \mathrm{mmm}$ with the lattice parameters of the tetragonal unit cell being $a=0.3884 \mathrm{~nm}$ and $c=1.9272 \mathrm{~nm}$. Good agreement between the calculated and observed positions and intensities of the lines indicates that this sample is a single phase of the $\mathrm{Sr}_{3} \mathrm{Ti}_{2} \mathrm{O}_{7}$-type, not a mixture with the $\mathrm{ABO}_{3}$ and $\mathrm{K}_{2} \mathrm{NiF}_{4}$-type structure.

The temperature dependence of the resistance $R$ without field for $\mathrm{La}_{2-2 x} \mathrm{Ca}_{1+2 x} \mathrm{Mn}_{2} \mathrm{O}_{7}$ with $x=0.2$ is depicted in the top panel of Figure 3. As is evident that the $R-T$ profile shows a sharp cusp at $\sim 107 \mathrm{~K}$ $\left(T_{p}\right)$, with semiconductor behavior above and metallic behavior below this temperature. Within the semiconductor range, the resistance of $\mathrm{La}_{2-2 x} \mathrm{Ca}_{1+2 x} \mathrm{Mn}_{2} \mathrm{O}_{7}$ falls steadily with increasing temperature, following 
the law $R \sim \exp \left(T_{0} / T\right)^{\nu}$ with $\nu \simeq 1 / 4 . \nu=1 / 4$ corresponds to Mott's expression for variable-range hopping of charge carriers in a band of localized states in the absence of electron correlations [14].

The bottom panel of Fig. 3 shows the temperature dependence of the MR under an applied field of 0.1 Tesla. The MR at $10 \mathrm{~K}$ becomes as high as $25 \%$. It is clear that the MR shows a gradual decline with increasing temperature below near $T_{p}$, followed by an abrupt decrease above this temperature. No MR peaks were observed. This kind of temperature profile of MR is reminiscent of that found in heteroepitaxial magnetic tunneling junctions containing magnetic electrodes of manganites separated by an insulating barrier layer [10]. Their temperature profiles of MR are very similar.

To understand this MR behavior it seems essential to consider the structure of the sample. As mentioned above, the layered perovskite crystals are a stack of ferromagnetic-metallic (FM) sheets composed of the $\mathrm{MnO}_{2}$ bilayers which are separated by insulating ( $\mathrm{La}$, $\mathrm{Ca})_{2} \mathrm{O}_{2}$ layers and form a natural array of FM / Insulator / FM junctions. So do the polycrystals within a grain. Previous studies showed that at low temperatures $\left(T<T_{c}\right)$ the moments of the $\mathrm{MnO}_{2}$ bilayers are essentially parallel within domains separated by domain boundaries lying on the (La, Ca) $)_{2} \mathrm{O}_{2}$ layers [12]. Recent experiments indicate that the low-temperature phase of this system may consist of mostly antiferromagnetic and some ferromagnetic static order between the adjacent $\mathrm{MnO}_{2}$ bilayers [13, 15]. In this sense, a relatively low field can easily align the moment of the adjacent $\mathrm{MnO}_{2}$ and makes it more easy that nearly fully spin-polarized carriers tunnel through the insulating $(\mathrm{La}, \mathrm{Ca})_{2} \mathrm{O}_{2}$ layers between the adjacent $\mathrm{MnO}_{2}$ bilayers, giving a large low-field tunneling $\mathrm{MR}$ which increases with decreasing temperature. At temperature $\left(T \geq T_{c}\right.$ ), the magnetic ordering alongthe $c$-direction is destroyed. A low field (such as a 0.1 Tesla field) can not align the $c$-direction spins, so the MR in these temperature ranges are very small.

In Fig. 4, we display the normalized isothermal MR and Magnetization (M) as a function of the applied field at a temperature of $10 \mathrm{~K}$. Resistance shows an abrupt decrease in the low-field range and becomes constant when the $\mathrm{M}$ is saturated under applied fields of about 0.3 Tesla. This confirms the conclusion that a relatively low field can easily align the moment of the adjacent $\mathrm{MnO}_{2}$, thus giving a large low-field tunneling MR at low temperature. As is evident, the

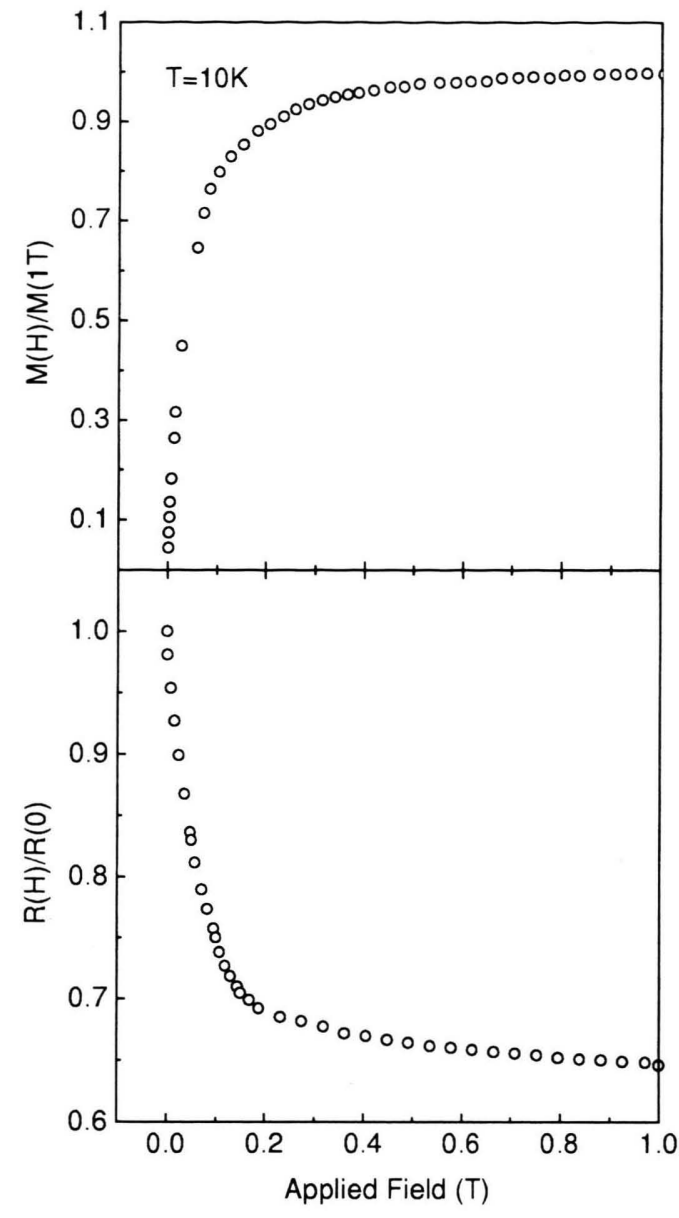

Fig. 4. The normalized isothermal MR and Magnetization (M) as a function of applied field at the temperature of $10 \mathrm{~K}$.

sensitivity of the MR response to the field is high in the low-field range.

Because of the polycrystalline nature of the sample, the grain-boundary transport also plays a role in the low-field MR at low temperature. That is to say the spin-polarized tunneling between grains [11] may be another factor which causes the high low-field MR at low temperature in the layered $\mathrm{La}_{2-2 x} \mathrm{Ca}_{1+2 x} \mathrm{Mn}_{2} \mathrm{O}_{7}$ polycrystal, although currently its weight can not be known.

The low-field MR of polycrystalline $\mathrm{La}_{1-x} \mathrm{Ca}_{x}$ $\mathrm{MnO}_{3}(x=0.2)$, the perovskite parent compound of two-layered $\mathrm{La}_{2-2 x} \mathrm{Ca}_{1+2 x} \mathrm{Mn}_{2} \mathrm{O}_{7}$ was also measured at $10 \mathrm{~K}$ for comparison: $\mathrm{MR} \sim-4 \%,-6.5 \%$ and $8 \%$ under applied fields of $0.05,0.1$ and 0.15 Tesla, respectively. These values are significantly smaller than those $(-17 \%,-25 \%$ and $-30 \%$ under applied 
fields of $0.05,0.1$ and 0.15 Tesla, respectively) for the two-layered $\mathrm{La}_{2-2 x} \mathrm{Ca}_{1+2 x} \mathrm{Mn}_{2} \mathrm{O}_{7}$. The $\mathrm{MR}$ value of $-30 \%$ under an applied of 0.15 Tesla is also larger than that $(\sim-12 \%$ under 0.15 Tesla field at $4.2 \mathrm{~K}$ ) observed in polycrystalline $\mathrm{La}_{1-x} \mathrm{Ca}_{x} \mathrm{MnO}_{3}$ films [16]. However, the low-field MR values in twolayered $\mathrm{La}_{2-2 x} \mathrm{Ca}_{1+2 x} \mathrm{Mn}_{2} \mathrm{O}_{7}$ polycrystal are smaller than those found in heteroepitaxial magnetic tunneling junction containing magnetic electrodes of the manganites separated by an insulating barrier layer [10] and two-layered single crystals [12, 13]. This

[1] M. N. Baibich, J. M. Broto, A. Fert, F. Nguyen Van Dau, F. Petroff, P. Etienne, G. Creuzet, A. Friederich, and J. Chaezelas, Phys. Rev. Lett. 61, 2472 (1988).

[2] G. Binasch, P. Grünberg, F. Sauerbach, and W. Zinn, Phys. Rev. B 39, 4828 (1989).

[3] J. Q. Xiao, J. S. Jiang, and C. L. Chien, Phys. Rev. Lett. 68, 3749 (1992).

[4] A. E. Berkowitz, J. R. Mitchel, M. J. Carey, A. P. Young, S. Zhang, F. E. Spada, F. T. Parker, A. Hutten, and G. Thomas, Phys. Rev. Lett. 68, 3745 (1992).

[5] B. Dieny, V. S. Speriosu, S. S. P. Parkin, B. A. Gurney, D. R. Wilhoit, and D. Mauri, Phys. Rev. B 43, 1279 (1991).

[6] T. Miyazaki and N. Tezuka, J. Magn. Magn. Mater. 139, L231 (1995).

[7] J. S. Moodera, L. R. Kinder, T. M. Wong, and R. Meservey, Phys. Rev. Lett. 74, 3237 (1995).

[8] J. M. Coey, A. E. Berkowitz, Ll. Balcells, F. F. Putris, and A. Barry, Phys. Rev. Lett. 80, 3815 (1998). may be the reason why much more impurities and defects are found in the former case, while no or few are found in the two latter cases. These impurities and defects can enhance the spin-flip effects of the tunneling carriers and reduce the low-field MR in polycrystals [17].

In short, by the sol-gel method a polycrystal bulk of two-layered $\mathrm{La}_{2-2 x} \mathrm{Ca}_{1+2 x} \mathrm{Mn}_{2} \mathrm{O}_{7}$ with $x=0.2$ was successfully synthesized, and a high sensitive lowfield TMR was discovered at low temperature.

This work is supported by NMS and NSFC.

[9] S. Lee, H. Y. Hwang, Boris I. Shraiman, W. D. Ratcliff II, and S-W. Cheong, Phys. Rev. Lett. 82, 4508 (1999).

[10] Y. Lu, X. W. Li, G. Q. Gong, G. Xiao, A. Gupta, P. Lecouer, J.Z. Sun, Y. Y. Wang, and V.P. Dravid, Phys. Rev. B 54, R8357 (1996).

[11] H. Y. Hwang, S-W. Cheong, N. P. Ong, and B. Batlogg, Phys. Rev. Lett. 77, 2041 (1996).

[12] T. Kimura, Y. Tomioka, H. Kuwahara, A. Asamitsu, M. Tamura, and Y. Tokura, Science 274, 1698 (1996).

[13] T. Kimura, A. Asamitsu, Y. Tomioka, and Y. Tokura, Phys. Rev. Lett. 79, 3720 (1997).

[14] N. F. Mott, Adv. Phys. 21, 785 (1972).

[15] T. G. Perring, G. Aeppli, T. Kimura, Y. Tokura, and M. A. Adams, Phys. Rev. B 58, R14693 (1998).

[16] X. W. Li, A. Gupta, G. Xiao, and G. Q. Gong, Appl. Phys. Lett. 71, 1124 (1997).

[17] P. Lyu, D. Y. Xing, and J. Dong, Phys. Rev. B 58, 54 (1998). 